INPLASY

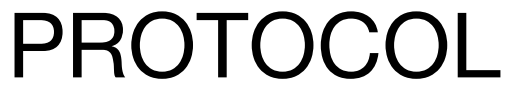

To cite: Wang et al. Effect of traditional Chinese exercise on abnormal lipid metabolism in patients with atherosclerosis. Inplasy protocol 202190093. doi:

10.37766/inplasy2021.9.0093

Received: 25 September 2021

Published: 25 September 2021

Corresponding author:

Chen Wang

qingshen126@126.com

Author Affiliation:

The School of Sports Medicine and Rehabilitation, Beijing

University of Sports, China.

Support: National Sports

Administration.

Review Stage at time of this submission: Piloting of the study selection process.

Conflicts of interest:

None declared.

\section{Effect of traditional Chinese exercise on abnormal lipid metabolism in patients with atherosclerosis}

\author{
Wang, X1; Zhang, P2; Yang, Y33.
}

Review question / Objective: The aim of this meta-analysis was to observe the effect of traditional Chinese exercise on abnormalities in lipid metabolism in patients with atherosclerosis.

Condition being studied: Atherosclerosis. Randomized clinical trials will be included irrespective of blinding, publication status or language.

Information sources: The two authors (CW and PZZ) extracted the data independently using Microsoft Excel. Any disagreements that arose during the extraction of the data were to be resolved by negotiation. Reconciliation of the data was done by a third independent person. We extracted study design, participant demographics, intervention details and means and standard deviations of all outcomes. Where necessary, we searched published protocols and trial registries for further methodological details and risk of bias assessments. If any of the included studies were found to have missing data, the first author of the paper and corresponding author were contacted by E-mail to obtain missing data to complete the meta-analysis.

INPLASY registration number: This protocol was registered with the International Platform of Registered Systematic Review and Meta-Analysis Protocols (INPLASY) on 25 September 2021 and was last updated on 25 September 2021 (registration number INPLASY202190093).

\section{INTRODUCTION}

Review question / Objective: The aim of this meta-analysis was to observe the effect of traditional Chinese exercise on abnormalities in lipid metabolism in patients with atherosclerosis.

Condition being studied: Atherosclerosis. Randomized clinical trials will be included 
irrespective of blinding, publication status or language.

\section{METHODS}

Search strategy: S1 ( MH("Random Assignment" OR "Placebos" OR "Placebo Effect" OR "Single-Blind Studies" OR "Double-Blind Studies" OR "Triple-Blind Studies" OR "Randomized Controlled Trials" OR "comparative studies" OR "Evaluation Research" OR "Prospective Studies" OR "crossover Design" OR "Prospective Studies" OR "Clinical Trials" OR "Clinical Trial Registry") ) OR ( TX(random\$ OR allocation OR "random allocation" OR placebo\$ OR single blind OR double blind OR "randomi?ed controlled trial*" OR "controlled clinical trial*" OR "comparative study" OR "evaluation stud*" OR "follow-up stud*" OR "prospective stud*" OR "cross-over stud*" OR control\$ OR prospectiv\$ OR volunteer\$ OR "RCT" OR "clinical trial*") ) OR ( PT( randomized controlled trial OR "clinical trial*") ) | S2 TX("Arteriosclerosis" OR "Atherosclerosis" OR "Peripheral Arterial Disease") | S3 AB(tai chi OR taiji OR qigong OR liuzijue OR wuqinxi OR yijinjing OR baduanjin OR traditional exercise OR chinese traditional exercise OR traditional chinese exercise OR chinese exercise) | S4 S1 AND S2 AND S3.

Participant or population: Inclusion: We will include studies which participants over 18 years old, either sex, have been diagnosed with atherosclerosis (confirmed by ultrasound, arteriography or other medical check) or evaluated with high risk of atherosclerosis (old age, high lipid profile, rheumatoid arthritis, etc.). Exclusion: Subjects who had any serious diseases (e.g., cardiovascular disease, sequela of apoplexy), a history of drug, or alcohol abuse were excluded. And we will exclude studies which intervention or control group involved other exercise therapies like yoga. Also the studies with incomplete outcome data and not available after contacting the authors will be exclude.

Intervention: Traditional Chinese exercises (e.g. Tai Chi Chuan, Five Animal Play, Six
Characters, Yi Jin Jing and Eight Duan Jin) will be the main intervention or as an addon therapy with a training period of more than 3 months.

Comparator: A group of patients who were not received traditional Chinese exercise therapy? including but not limited to wait list, conventional exercise and so on.

Study designs to be included: We will include studies which participants over 18 years old, either sex, have been diagnosed with atherosclerosis (confirmed by ultrasound, arteriography or other medical check) or evaluated with high risk of atherosclerosis (old age, high lipid profile, rheumatoid arthritis, etc.).

Eligibility criteria: Eligibility criteriaType of participant. Patients with a diagnosis of atherosclerosis. Inclusion criteria: (1) Meeting the diagnostic criteria for carotid atherosclerosis; (2) Complete clinical information; (3) Informed consent. Exclusion criteria: (1) Those with co-morbid heart, brain, liver, kidney and lung diseases. (2) Those who have had a cerebrovascular accident in the 6 months prior to enrolments; (3) Those with psychiatric disorders.Interventions. We included all traditional Chinese gong methods (Taijiquan, Qigong, Five Animals, Six Characters, Eight Duan Jin) and excluded medication that had been shown to have no significant therapeutic effect or to pose a significant safety risk.Type of control group. The control group can be either intreatment or standard treatment, as long as it does not receive specific complementary and alternative therapies corresponding to the experimental group.Results. (1) $5 \mathrm{~mL}$ of peripheral fasting venous blood was collected from patients before and after treatment, and the levels of lipid indexes total cholesterol (TC), triacylglycerol (TG), low-density lipoprotein cholesterol (LDL C) and high-density lipoprotein cholesterol (HDL - C) were measured using the MVIS2035 fully automated blood rheology analyser. The main focus was to observe the effect of traditional Chinese gongfu on lipid metabolism in patients with atherosclerosis. 
Information sources: The two authors (CW and PZZ) extracted the data independently using Microsoft Excel. Any disagreements that arose during the extraction of the data were to be resolved by negotiation. Reconciliation of the data was done by a third independent person. We extracted study design, participant demographics, intervention details and means and standard deviations of all outcomes. Where necessary, we searched published protocols and trial registries for further methodological details and risk of bias assessments. If any of the included studies were found to have missing data, the first author of the paper and corresponding author were contacted by E-mail to obtain missing data to complete the metaanalysis.

Main outcome(s): total cholesterol(TC); triglyceride(TG); high-density lipoprotein cholesterol(HDL-C); low-density lipoprotein cholesterol(LDL-C)); Adiponectin (ADP).

Quality assessment / Risk of bias analysis: Assessing the risk of bias will be performed independently by two reviewers, using the Cochrane risk of bias tool. If any disagreement exist, discussing with the third viewer to solve it. The following characteristics of the studies will be assessed, methods of randomization, treatment allocation, blinding, incomplete outcome data, selective reporting, other sources of bias.

Strategy of data synthesis: Concentration values of outcome were collated in a same scale. Change in post-intervention mean was calculated by subtracting the baseline from follow-up values. When data on standard deviation (SD) were not available after contacting the authors, calculate from available data and imputation was used to approach it. Results were presented as weighted mean difference (WMD) with 95\% confidence interval (Cl). Higgins' $\mathrm{I}^{2}$ and $\mathrm{p}$ value of the Cochrane $Q$ statistics will be calculated to assess statistical heterogeneity. A random-effects model (REM) or a fixed-effects model (FEM) was applied when the heterogeneity was notable or not.
Subgroup analysis: We will include 2 types of participants, which involved people with high risk of atherosclerosis and patients with atherosclerosis. The type of participants may have an impact on the intervention results. Divided the included studies into different subgroup according to different type of participants. Performing a meta-regression if the condition allows.

Sensitivity analysis: For results with large heterogeneity, we will use sensitivity analysis methods, such as using funnel plots to observe whether the included studies are symmetrical or using the egg's test in stata to observe sources of heterogeneity, and for data with large heterogeneity, subtractive complementary methods may be used. This can also be done using meta-regression methods.

\section{Language: English.}

Country(ies) involved: China.

Keywords: traditional Chinese exercise, Atherosclerosis, lipid metabolism, metaanalysis.

Contributions of each author:

Author 1 - Chen Wang.

Author 2 - Peizheng Zhang.

Author 3 - Yuting Yang. 